\title{
Article
}

\section{Controlling Self-Assembling Peptide Hydrogel Properties through Network Topology}

Gao, Jie, Tang, Claire, Elsawy, Mohamed, Smith, Andrew M., Miller, Aline F. and Saiani, Alberto

Available at https://clok.uclan.ac.uk/18414/

Gao, Jie, Tang, Claire, Elsawy, Mohamed orcid iconORCID: 0000-0003-39642150, Smith, Andrew M., Miller, Aline F. and Saiani, Alberto (2017) Controlling Self-Assembling Peptide Hydrogel Properties through Network Topology.

Biomacromolecules, 18 (3). pp. 826-834. ISSN 1525-7797

It is advisable to refer to the publisher's version if you intend to cite from the work.

http://dx.doi.org/10.1021/acs.biomac.6b01693

For more information about UCLan's research in this area go to

http://www.uclan.ac.uk/researchgroups/ and search for <name of research Group>.

For information about Research generally at UCLan please go to http://www.uclan.ac.uk/research/

All outputs in CLoK are protected by Intellectual Property Rights law, including Copyright law. Copyright, IPR and Moral Rights for the works on this site are retained by the individual authors and/or other copyright owners. Terms and conditions for use of this material are defined in the policies page.

\section{CLoK}

Central Lancashire online Knowledge www.clok.uclan.ac.uk

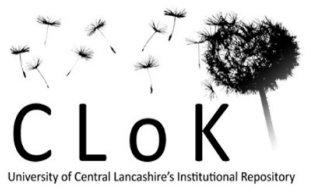




\title{
Controlling Self-Assembling Peptide Hydrogel Properties through ${ }_{2}$ Network Topology
}

\author{
3 Jie Gao, ${ }^{\dagger, \ddagger, \S}$ Claire Tang, ${ }^{\dagger, \ddagger}$ Mohamed Elsawy, ${ }^{\dagger, \ddagger}$ Andrew M. Smith, ${ }^{\dagger, \ddagger}$ Aline F. Miller, ${ }^{\ddagger}, \|$ \\ 4 and Alberto Saiani ${ }^{*}, \dagger,+(0)$
}

$5{ }^{\dagger}$ School of Materials, ${ }^{\ddagger}$ Manchester Institute of Biotechnology, and "School of Chemical Engineering and Analytical Sciences, The

6 University of Manchester, Oxford Road, Manchester, M13 9PL, United Kingdom

\section{S Supporting Information}

8 ABSTRACT: Self-assembling peptide-based hydrogels have encountered increasing interest in the recent years as scaffolds for 3D cell culture or for controlled drug delivery. One of the main challenges is the fine control of the mechanical properties of these materials. The bulk properties of hydrogels not only depend on the intrinsic properties of the fibers but also on the network topology formed. In this work we show how fiberfiber interactions can be manipulated by design to control the final hydrogel network topology and therefore control the final
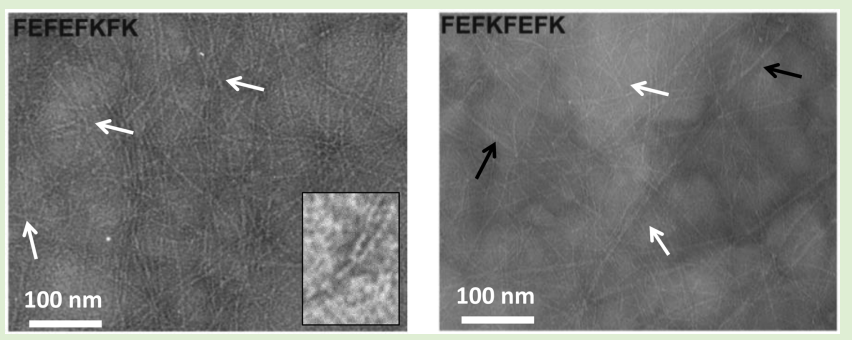
properties of the material. This was achieved by exploiting the design features of $\beta$-sheet forming peptides based on hydrophobic and hydrophilic residue alternation and exploiting the ability of the arginine's guanidine side group to interact with itself and with other amino acid side groups. By designing octa-peptides based on phenylalanine, glutamic acid, lysine, and arginine, we have investigated how fiber association and bundling affect the dynamic shear modulus of hydrogels and how it can be controlled by design. This work opens the possibility to fine-tune by design the bulk properties of peptide hydrogels.

24 The use of noncovalent self-assembly to construct materials has 25 become a prominent strategy in material science offering 26 practical routes for the construction of increasingly functional 27 materials for a variety of applications ranging from electronics 28 to biotechnology. ${ }^{1-4}$ A variety of molecular building blocks can 29 be used for this purpose; one such block that has attracted 30 considerable attention in the last 20 years is de novo designed 31 self-assembling peptides. ${ }^{5-8}$ One particular class of materials 32 with significant potential in the biological and biomedical fields 33 are self-assembling peptide based hydrogels. These highly 34 hydrated, biodegradable and biocompatible "soft" materials are 35 very attractive for the design of scaffolds for the $3 \mathrm{D}$ culture of 36 cells $^{9-11}$ and delivery of drugs. ${ }^{12,13}$ A number of self-assembling 37 peptide designs have emerged in the literature that allow the 38 fabrication of very stable hydrogels. One of the main challenges 39 that remain in the field is the fine-tuning of the mechanical 40 properties of these hydrogels as different cell types, and 41 therapeutic approaches require hydrogels with different proper42 ties.

43 The formation of hydrogels by self-assembling peptides 44 involves two distinct processes; the self-assembly of the 45 peptides themselves to form thin fibrillar structures and the 46 entanglement and association of these fibrils into a three47 dimensional percolated network (Figure 1). Developing a 48 fundamental understanding of these two processes at all length 49 scales is crucial as the properties of the final materials will not 50 only depend on the intrinsic properties of the fibers, but also on how they assemble and ultimately on the properties of the 51 network formed. Most of the focus in the literature is on the 52 first process, the self-assembly of the peptides into fibers. The 53 second process has been less studied and is less well 54 understood and is the focus of this work.

55

We have investigated in recent years the self-assembly of a 56 family of amphipathic short $\beta$-sheet forming peptides inspired 57 from Zhang's group work. ${ }^{14-20}$ These peptides are typically $8-58$ 16 residues long and their design is based on the alternation of 59 hydrophobic and hydrophilic amino acids. These peptides are 60 well-known to readily self-assemble into antiparallel $\beta$-sheets 61 and form, above a critical gelation concentration (CGC), 62 hydrogels that have been shown to support the growth of a 63 variety of cells, ${ }^{21-27}$ as well as allow the controlled delivery of a 64 variety of drugs. ${ }^{12,28-31}$ One interesting aspect of this design is 65 that formation of these sheets results in all the hydrophobic 66 side groups being located on the same face of the sheet, while 67 all the hydrophilic side groups are located on the opposite 68 face. $^{32-35}$ As a result it is speculated that two of these sheets 69 associate through their hydrophobic faces to form a fiber. 70 Above the CGC these fibers entangle and/or associate to form 71 a 3D percolated network that traps water, that is, a hydrogel 72 (Figure 1). As a consequence interfiber interactions which play 73

Received: November 15, 2016

Revised: January 6, 2017

Published: January 9, 2017 
A
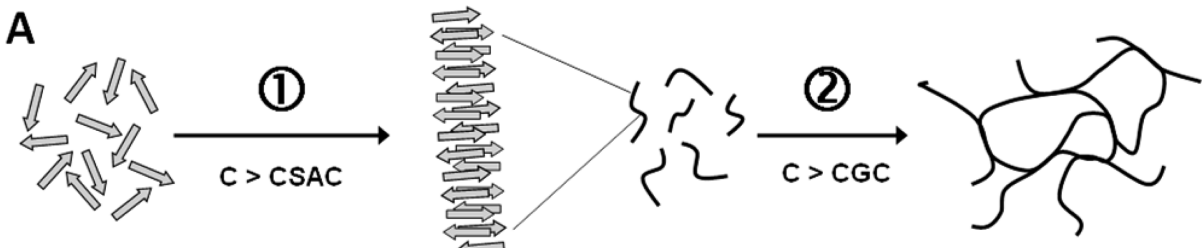

$\Longrightarrow \beta$-sheet forming peptide
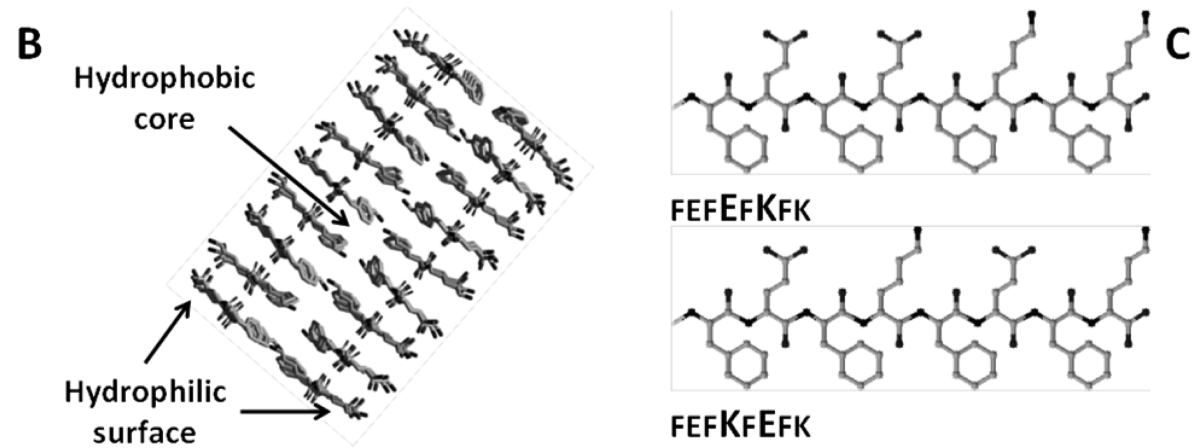

Figure 1. (A) Schematic representation of the self-assembly and gelation process of $\beta$-sheet forming peptides. (B) Schematic representation of $\beta$ sheet fibers. (C) Chemical structure of lysine containing peptides.

74 a key role in the fibrillar network formation are mainly 75 controlled by the hydrophilic residues.

76 In this work we were interested in understanding how 77 network topology affects the mechanical properties of the 78 hydrogel and how it can be used to design materials with 79 tailored properties. For this purpose we decided to use a family 80 of octapeptides based on the same design that are known to 81 self-assemble into $\beta$-sheet rich fibrils. This approach allowed us 82 to keep the fiber structure identical across all the systems and 83 focus on the effect of network topology on the mechanical 84 properties of the hydrogels formed. As mentioned above due to 85 the design chosen interfiber interactions are controlled mainly 86 by the hydrophilic residues. Of particular interest to us was 87 arginine, which is a residue that has a guanidine side group. 88 This side group can interact strongly with other amino acid side 89 groups as well as with itself in a variety of configurations, 90 including through hydrogen bonding and electrostatic inter91 actions, as well as salt bridges. ${ }^{36-38}$ Our hypothesis was that the 92 introduction of this amino acid would result in increased fiber93 fiber interactions and therefore would affect the topology of the 94 network formed and the final mechanical properties of the 95 hydrogels. For this purpose a family of phenylalanine based 96 peptides containing lysine $(\mathrm{K})$ and arginine $(\mathrm{R})$ were designed. 97 Hydrogels were prepared and characterized using a range of 98 techniques including Fourier transform infrared spectroscopy 99 (FTIR), small angle neutron scattering (SANS), transmission 100 electron microscopy (TEM), and oscillatory shear rheology.

\section{MATERIALS AND METHODS}

102 Materials. Peptides were purchased as TFA salts from Cambridge 103 Research Biochemicals (U.K.; FEFEFKFK and FEFKFEFK) and 104 Biomatik (U.S.A.; FEFEFRFK and FEFEFRFR) and used without 105 further purification. The purity of the compounds was verified by 106 HPLC (>95\%; see Supporting Information (SI) for HPLC traces) and 107 mass spectrometry. HPLC grade and deuterated (99.9 atom \% D) 108 water were purchased from Sigma-Aldrich (U.K.).

109 Sample Preparation. Depending on the desired formulation and 110 concentration the required amount of peptide(s) powders (or powder mixtures) was suspended in HPLC grade water. The samples were 111 vortexed and sonicated (VWR ultrasonicator bath, $30 \mathrm{~W}$ ) until the 112 peptide(s) was fully dissolved. Due to presence of TFA (typically 3:1 113 TFA to peptide ratio as the TFA is bound to the two cationic residues 114 as well as the terminal $\mathrm{NH}_{3}{ }^{+}$end group), the $\mathrm{pH}$ of the sample was 115 2.3-2.8. In order to have a common $\mathrm{pH}$ for all the samples, their $\mathrm{pH} 116$ was adjusted to 3.0 using a $1 \mathrm{M}$ solution of $\mathrm{NaOH}$ (typically $\sim 10-20117$ $\mu \mathrm{L}$ added, depending on starting $\mathrm{pH}$ ). The samples were then stored 118 at room temperature overnight, and the $\mathrm{pH}$ was checked again before 119 use.

Phase Diagram. The samples were prepared in a test tube as 121 described above and placed in a water bath, the temperature of which 122 was controlled by both mercury and Grant analogue thermometers. 123 The temperature of the bath was increased from 25 to $90{ }^{\circ} \mathrm{C}$ by steps 124 of $5{ }^{\circ} \mathrm{C}$. The samples were left to equilibrate at each temperature for 125 15 min before their macroscopic state was assessed through the "test- 126 tube tilting" method.

127

Fourier Transform Infrared Spectroscopy (FTIR). Multiple 128 bounce attenuated total reflectance (ATR) FTIR experiments were 129 undertaken using samples prepared in water. Spectra were recorded on 130 a Thermo Nicolet 5700 spectrometer equipped with a trough plate 131 comprising of a zinc selenide crystal, which permitted 12 reflections 132 with a $45^{\circ}$ angle of incidence. The samples were spread directly on the 133 surface of the trough plate. Spectra were acquired in the 4000-400 134 $\mathrm{cm}^{-1}$ range with a resolution of $4 \mathrm{~cm}^{-1}$ over 256 scans. The water 135 spectrum was used as background and subtracted from all spectra 136 using Omnic software (version 7.2, Thermo Electron Corporation) 137 provided with the instrument.

Dynamic Oscillatory Shear Rheometry. Viscoelastic properties 139 were assessed in an oscillatory mode, using a stress-controlled 140 rheometer (TA Instruments AR-G2) equipped with a Peltier plate 141 to control temperature. A parallel plate geometry was used with a 142 diameter of $20 \mathrm{~mm}$ and a gap of $250 \mu \mathrm{m}$. To ensure the measurements 143 were made in the linear regime, amplitude sweeps were performed and 144 showed no variation in $G^{\prime}$ and $G^{\prime \prime}$ up to a strain of $1 \%$. The dynamic 145 shear moduli of the hydrogel were measured at $1 \mathrm{~Hz}$ with a strain of 146 $0.1 \%$. All experiments were performed at $25{ }^{\circ} \mathrm{C}$ at least three times to 147 ensure reproducibility.

148

Transmission Electron Microscopy (TEM). Samples were 149 prepared at $10 \mathrm{mg} \mathrm{mL}^{-1}$ and diluted 10-fold. The solutions were 150 vortexed until they were fully homogeneous. A total of $20 \mu \mathrm{L}$ of 151 sample was adsorbed onto the glow-discharged, carbon-coated copper 152 

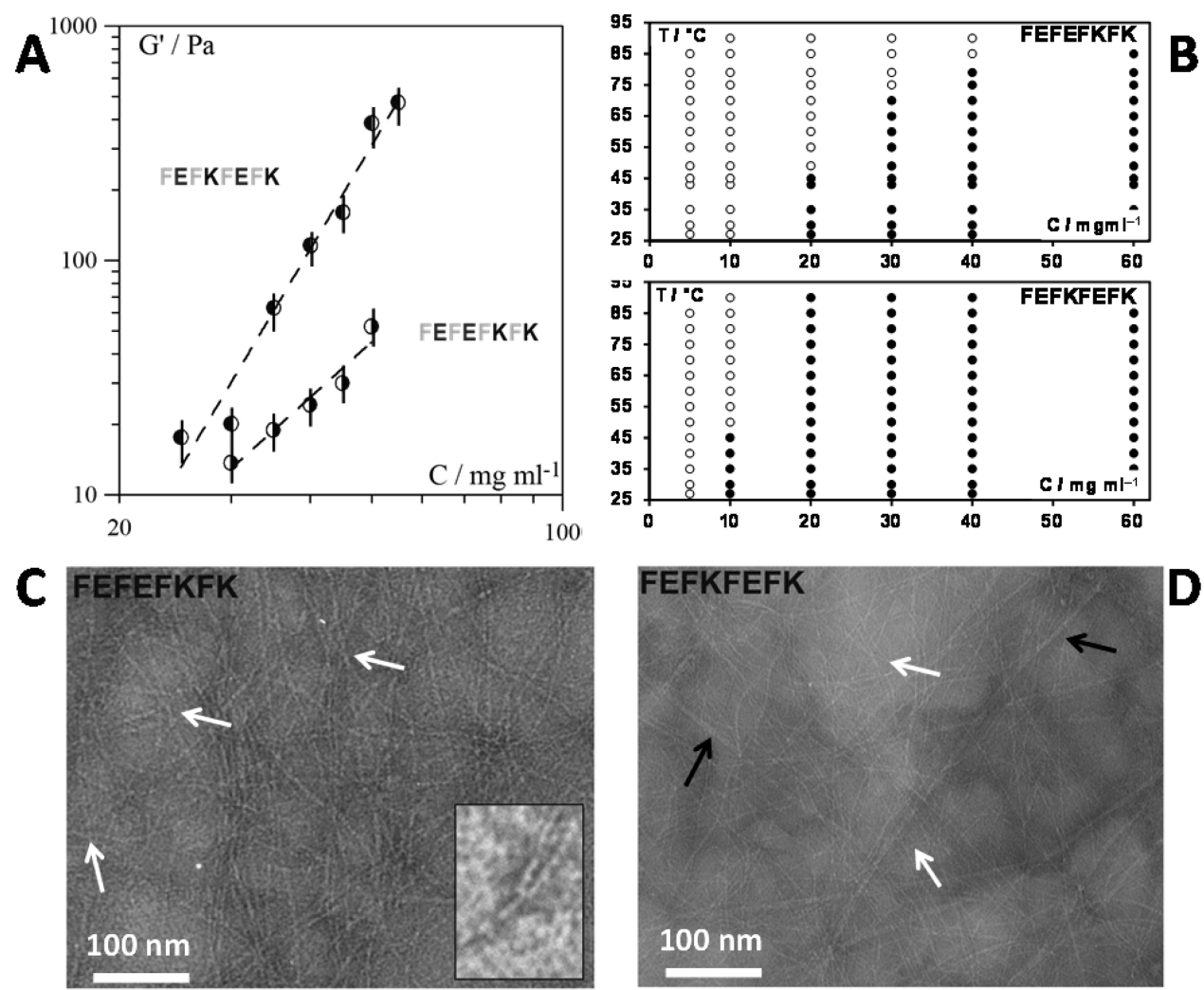

Figure 2. (A) Dynamic shear moduli of lysine based peptide hydrogels (see Figure 1 for chemical structures). (B) Temperature vs concentration phase diagrams (open symbols: liquid/viscous liquid and closed symbols weak gels/gels). (C, D) TEM images obtained for FEFEFKFK (C: inset, high resolution image showing details of a "Y" junction) and FEFKFEFK samples (D). White arrows show "Y" junction point, while black arrows show associated fibers. For more information, see text.

153 grid (400 mesh, Agar Scientific) for 30 s. The loaded grids were 154 washed in distilled water for $15 \mathrm{~s}$ and negatively stained with $20 \mu \mathrm{L}$ of $1551 \%(\mathrm{w} / \mathrm{v})$ uranyl acetate. The grids were then blotted on Whatman 50 156 filter paper and allowed to air-dry for $30 \mathrm{~min}$ prior to observation. Data 157 was collected at high vacuum on a FEI Tecnail2 BioTwin transmission 158 electron microscope connected to a high resolution Orius CCD 159 SC1000 camera.

160 Small Angle Neutron Scattering (SANS). SANS experiments 161 were performed at the Forshungszentrum Jülich (FRJ-2) on 162 diffractometer KWS-2. The white beam was monochromated with a 163 velocity selector by Dornier. The neutron wavelength was $\lambda=0.48 \mathrm{~nm}$ 164 with a wavelength distribution characterized by a full width at half165 maximum $\Delta \lambda / \lambda=0.1$. Samples were irradiated on an area of $0.8 \times 0.8$ $166 \mathrm{~cm}^{2}$. The beamline was equipped with a two-dimensional detector with 167 a $50 \times 50 \mathrm{~cm}^{2}$ active area with a spatial resolution of $0.8 \times 0.8 \mathrm{~cm}^{2}$ 168 (further details are available on request at Forshungszentrum Jülich). 169 By varying the sample-detector distance, the available momentum 170 transfer vector $(q)$ was in the range of $0.1<q\left(\mathrm{~nm}^{-1}\right)<2.4$, with $q=$ $171(4 \pi / \lambda) \sin (\theta / 2)$, where $\theta$ is the scattering angle. The collected data 172 was corrected for the detector efficiency and dark current background. 173 Counter normalization was achieved by using the incoherent scattering 174 of an amorphous hydrogenous poly(methyl methacrylate) secondary 175 standard. After ensuring the scattering was isotropic, the data were 176 radially averaged to obtain a one-dimensional scattering curve. Under 177 these conditions, the normalized intensity scattered by a sample is

178

$$
I_{\mathrm{N}}(q)=\left[\frac{I_{\mathrm{s}}(q)}{T_{\mathrm{s}} \delta_{\mathrm{s}}}-\frac{I_{\mathrm{e}}(q)}{T_{\mathrm{e}} \delta_{\mathrm{e}}}\right]
$$

179 where $I_{\mathrm{N}}(q), \delta$, and $T$ are the normalized measured intensity, the 180 thickness, and the transmission of the sample (s) and the empty cell 181 (e), respectively. To extract the coherent intensity scattered by the 182 peptides, we subtracted the coherent intensity scattered by the solvent 183 and the incoherent intensity scattered by the peptide and the solvent from the total scattered intensity. The coherent intensity scattered by 184 the peptides in absolute units is then

$$
I_{\mathrm{A}}(q)=\frac{1}{K}\left[I_{\mathrm{N}}(q)-\left(1-C_{\mathrm{p}}\right) I_{\mathrm{D}}(q)-I_{\mathrm{b}}\right]
$$

where $I_{\mathrm{D}}(q)$ is the normalized intensity scattered by the deuterated 187 solvent, $C_{\mathrm{P}}$ is the peptide concentration in $\mathrm{g} \mathrm{cm}^{-3}, I_{\mathrm{b}}$ is the background 188 scattering mainly due to the incoherent scattering of the hydrogenous 189 peptides, and $K$ is the contrast factor. The background scattering, $I_{\mathrm{b}}, 190$ was estimated using the Porod law, which gives the scattered intensity 191 of a two-phase system at high $q$ values: ${ }^{39-41}$

$$
I(q)=\frac{K_{\mathrm{p}}}{q^{4}}+I_{\mathrm{b}}
$$

where $K_{\mathrm{p}}$ is the Porod constant. $I_{\mathrm{b}}$ was estimated by fitting the last 10194 data points $\left(2.5-3.0 \mathrm{~nm}^{-1}\right)$ of the scattering curves, where the 195 background scattering is dominant, using a Porod representation 196 $\left(q^{4} I(q)\right.$ vs $\left.q^{4}\right)$. Solutions and hydrogel samples for neutron 197 experiments were prepared as described above using deuterated 198 water directly in HELLMA quartz cells with an optical path length of 2199 and $5 \mathrm{~mm}$, depending on the sample concentration. Deuterated water 200 $\left(\mathrm{D}_{2} \mathrm{O}\right)$ was used instead of hydrogenated water to increase the 201 contrast between the peptides and the solvent.

\section{RESULTS AND DISCUSSION}

In an earlier study we investigated the self-assembly of a series 204 of octa-peptides that included FEFEFKFK and FEFKFEFK (F: 205 phenylalanine; K: lysine; E: glutamic acid). These two peptides 206 differ only by the position of the central $\mathrm{E}$ and $\mathrm{K}$ residues 207 (Figure 1). In this earlier study, FTIR was used to show that 208 both peptides had the same tendency to form $\beta$-sheet fibers. In 209 addition, SANS experiments carried out at low concentration, 210 


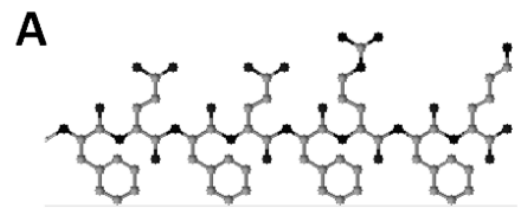

FEFEFRFK

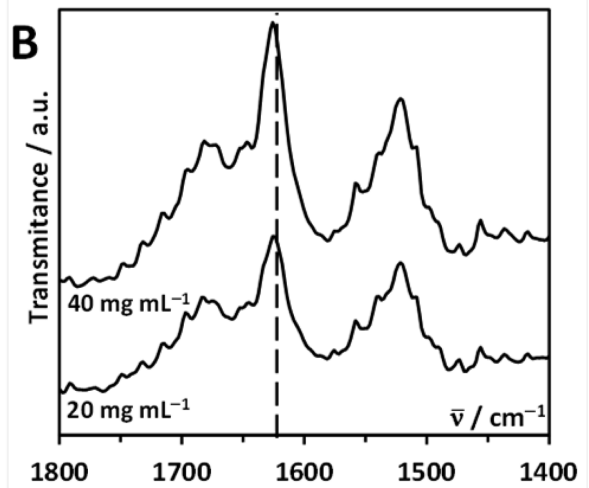

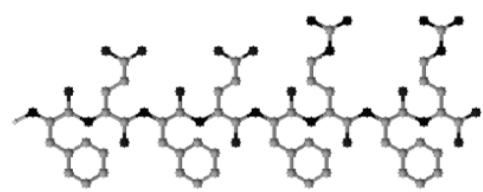

FEFEFRFR

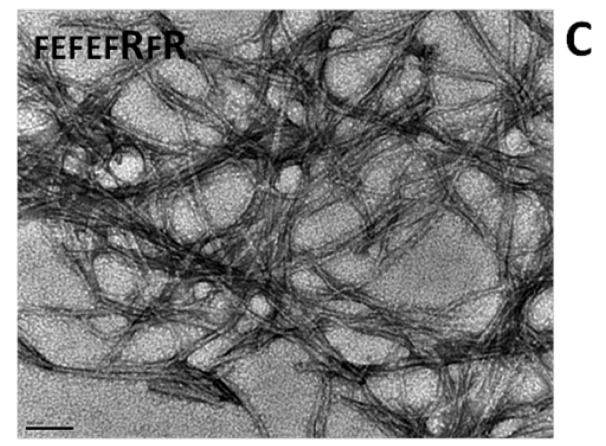

Figure 3. (A) Chemical structure of arginine (R) containing peptides. (B) FTIR spectra of FEFEFRFR sample at $5 \mathrm{mg} \mathrm{mL}^{-1}(\beta$-sheet band: 1624 $\mathrm{cm}^{-1}$ ). (C) TEM image obtained for FEFEFRFR sample (scale bar: $100 \mathrm{~nm}$ ).

211 that is, below the critical gelation concentration (CGC), 212 revealed that similar fibers were formed by both peptides. ${ }^{17}$ 213 One puzzling result obtained since that study has been the 214 difference in mechanical properties of the hydrogels formed. 215 Indeed, as can be seen from Figure 2A higher shear moduli, in 216 particular, at higher peptide concentrations were obtained for 217 FEFKFEFK. In addition, the temperature-concentration phase 218 diagrams of these two systems were also found to differ 219 significantly (Figure 2B). FEFKFEFK formed hydrogels at 220 lower concentration $\left(>5 \mathrm{mg} \mathrm{mL}^{-1}\right)$ that could not be melted, 221 while FEFEFKFK formed hydrogels at higher concentration $222\left(>10 \mathrm{mg} \mathrm{mL}^{-1}\right)$ that could be melted into viscous liquids at 223 high temperature. Based on our earlier work, our hypothesis 224 was that the differences in mechanical properties and phase 225 behavior were due to differences in network topologies rather 226 than differences in fiber intrinsic properties. TEM supported 227 this assumption. As can be seen from Figure 2C, TEM images 228 show the formation of a network of uniform thin entangled 229 fibers for FEFEFKFK with dimensions, 3-5 nm, which agrees 230 with our earlier work. "Y" shaped branching points can be 231 observed where one fiber "splits" into two fibers of the same 232 dimensions. The branching of $\beta$-sheet forming peptides was 233 discussed by Pochan and co-worker for a family of $\beta$-hairpin 234 peptides. These authors suggested that branching was due to 235 the mis-assembly of the peptides. ${ }^{42}$ Keeping in mind that each 236 fiber is formed by two $\beta$-sheets (Figure 1), it is reasonable to 237 assume a similar origin for the "Y" shaped branching observed 238 here. For FEFKFEFK, a slightly different network topology is 239 observed. Although "Y" shaped branching of fibers can still be 240 observed, thicker fiber bundles, formed from the association 241 along their length of two or more fibers, can also be seen 242 (Figure 2D). This suggests that the positions of $\mathrm{K}$ and $\mathrm{E}$ affects 243 the tendency of the fibers to interact. It should be noted that at $244 \mathrm{pH} 3$ these peptides and, therefore, the fibers they form carry a 245 positive charge and that the presence of TFA in the sample will 246 result in some level of charge screening that will promote 247 peptide self-assembly and sample gelation. The position of the 248 lysine residues affects the overall charge distribution on the fiber surface and therefore the way the electrostatic repulsion 249 acts. $^{43,44}$ The different topologies observed for these two 250 networks agree well with the observations above. Indeed, the 251 association of fibers along their length is expected to result in 252 stiffer fiber bundles and a stiffer network, resulting in a higher 253 shear modulus. Fiber bundling is expected to increase with 254 concentration, as fibers come into closer proximity; therefore, 255 the effect on the mechanical properties is also expected, as 256 observed, to be more marked at higher concentrations. In 257 addition, this association tendency is thought to also promote 258 the formation of a more stable percolated network resulting in a 259 lower CGC and a reduced melting tendency. Indeed, fiber 260 bundles are expected to be more difficult to melt/disassociate 261 than "Y" branching points as the latter can be considered weak 262 points.

It is well-known that hydrogel mechanical properties are very 264 sensitive to network topology. A number of theoretical models 265 can be found that describe the mechanical properties of 266 hydrogels. ${ }^{45}$ They usually relate the modulus of the hydrogel to 267 the concentration through power laws. One such model was 268 proposed by Jones and Marques for a network of semirigid 269 fibers joined at frozen junction points. ${ }^{46}$ It relates the shear 270 modulus of the hydrogel to the sample concentration through 271

$$
G^{\prime} \sim C^{(3+\mathrm{FD}) /(3-\mathrm{FD})}
$$

where FD is the fractal dimension of interconnecting objects. 273 For semirigid fibers, FD is expected to be in the range of 1.0- 274 1.2 corresponding to an exponent of $2.0-2.3$. The following 275 power laws were obtained for our two peptides:

$$
\text { FEFEFKFK } \Rightarrow G^{\prime} \sim C^{2.4} \text { and FEFKFEFK } \Rightarrow G^{\prime} \sim C^{4.6}
$$

A good agreement was obtained for the FEFEFKFK system 277 with Jones and Marques model. The network topology 278 observed for this system (uniform semirigid fibers connected 279 at frozen junction points) resembles the most the network 280 topology assumed in this model. For FEFKFEFK, a larger 281 exponent than predicted was obtained. There are a number of 282 reasons for the deviation from the model. First of all, the model 283 

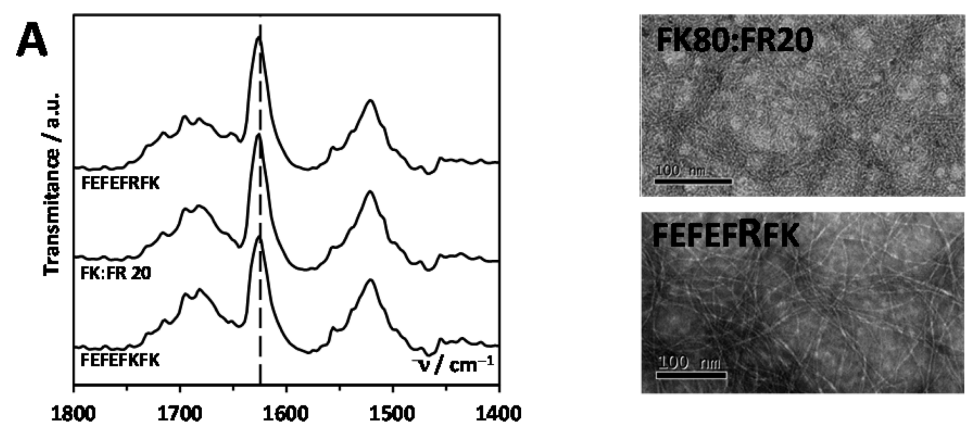

B
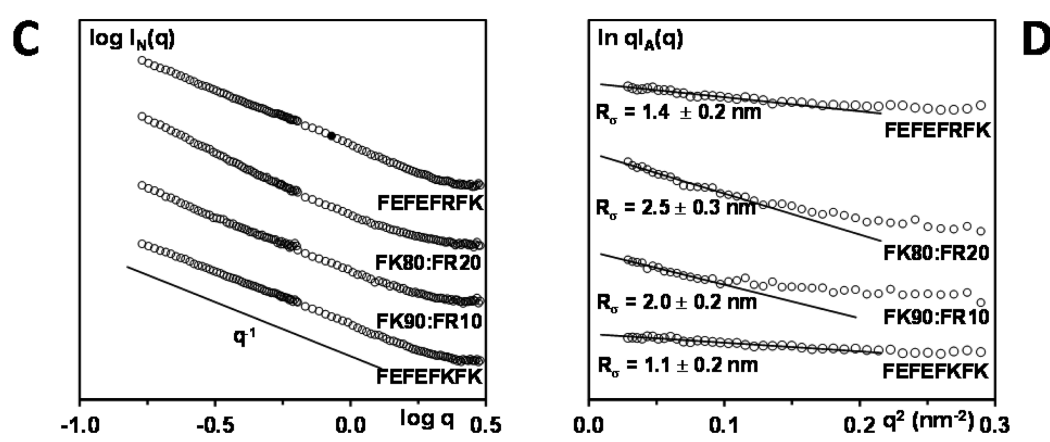

Figure 4. (A) FTIR spectra of hydrogels samples ( $\beta$-sheet band: $\left.1624 \mathrm{~cm}^{-1}\right)$; (B) TEM image obtained for hydrogel samples (scale bar: $100 \mathrm{~nm}$ ); (C) SANS scattering pattern $(\log I(q)$ vs $\log q)$ obtained for the sample below the CGC $\left(5 \mathrm{mg} \mathrm{mL}^{-1}\right)$; (D) SANS scattering pattern at low $q$ plotted in a $\ln \left[q I_{\mathrm{A}}(q)\right]$ vs $q^{2}$ representation. Best fits of curves linear sections are presented with the corresponding values of $R_{\sigma}$ extracted (see text for more details).

284 does not take into consideration fiber polydispersity (through 285 bundle formation) and, therefore, variation in network basic 286 element properties, such as stiffness. In addition, as the model 287 was developed for polymeric systems, the concentration is 288 assumed to directly relate to the concentration of connecting 289 elements in the network. As shown by Ramzi et al., the 290 presence in a polymeric network of pendant chains that do not 291 participate in the network elasticity can result in a higher than 292 expected exponent, as the "effective" network element 293 concentration is lower than the polymer concentration. ${ }^{47}$ In 294 our case, there is no direct evidence of the presence of pendant 295 chains. Nevertheless, the fiber thickening observed for 296 FEFKFEFK system is thought to have a similar effect. Indeed, 297 fiber bundles will act as a single element contributing to the 298 network elasticity, resulting in a lower apparent network 299 "effective" concentration and, therefore, in a higher exponent. 300 The results discussed above suggest that fiber association can 301 be used to control network topology and, therefore, the 302 mechanical properties of the peptide hydrogels. To confirm 303 that such an approach can be used to design hydrogels with 304 tailored properties, arginine residues were introduced in our 305 peptide design by replacing one or both of the lysines. As 306 mentioned in the introduction, arginine is expected to promote 307 through its guanidine side group strong fiber-fiber interactions. 308 The following two sequences were designed: FEFEFRFK 309 and FEFEFRFR (Figure 3A). For FEFEFRFR, opaque viscous 310 solutions/precipitates were obtained at all concentrations. 311 FTIR confirmed the adoption by this peptide of a $\beta$-sheet 312 conformation (Figure 3B), and TEM revealed the presence of 313 similar fibers as above (Figure $3 \mathrm{C}$ ). The fibers though were 314 observed to form, in this case, large bundles/aggregates, clearly 315 showing that, as hypothesized, $\mathrm{R}$ promotes strong fiber-fiber 316 interactions. To prevent the formation of such large scale 317 aggregates and their precipitation, we decided to "dope" 318 FEFEFKFK hydrogels with small amounts of FEFEFRFR, 10 and $20 \%$ (wt), keeping the overall sample peptide concen- 319 tration constant. These two samples will be referred to as 320 FK90:FR10 and FK80:FR20, respectively.

321

FK90:FR10 and FK80:FR20 were found to form slightly 322 "hazy" hydrogels, while FEFEFRFK was found to form clear 323 transparent hydrogels in the concentration range investigated. 324 As can be seen from Figure 4A, FTIR results suggest that the $325 \mathrm{f} 4$ introduction of arginine did not affect the peptides tendency to 326 form $\beta$-sheets; indeed, similar relative peak intensities in the 327 amide region were observed for all samples. The formation of 328 fibrous networks was confirmed by TEM (Figure 4B).

329

A first series of SANS experiments was performed below the 330 CGC of the samples in the dilute regime. In this regime, the 331 scattering observed is dominated by the form factor of the 332 diluted scattering entities. As can be seen from Figure 4C, all 333 the samples scattering patterns present $a \sim q^{-1}$ behavior at low 334 $q$ typical of the scattering of fibers. For infinitely long rod-like 335 structures, that is, semirigid fibers, in the $q$ range investigated, 336 the scattered intensity can be written as $39,48,49$ 337

$$
q^{2} I_{\mathrm{A}}(q)=\pi q C_{\mathrm{p}} \mu_{\mathrm{L}} f\left(q R_{\sigma}\right)+C s t
$$

where $\mu_{\mathrm{L}}$ is the mass per unit length of the rod in $\mathrm{g} \mathrm{mol}^{-1} \mathrm{~nm}^{-1}, 339$ $C_{\mathrm{p}}$ is the peptide concentration in $\mathrm{g} \mathrm{cm}^{-3}$, and $f\left(q R_{\sigma}\right)$ represents 340 the cross-section scattering, $R_{\sigma}$ being the cross-section radius of 341 gyration of the rod. Cst is a constant term taking into account 342 interscattering effects. For $q R_{\sigma}<1$, eq 5 reduces to ${ }^{39,48-50} 343$

$$
q I_{\mathrm{A}}(q)=\pi C_{\mathrm{p}} \mu_{\mathrm{L}} \exp \left(\frac{q^{2} R_{\sigma}^{2}}{2}\right)
$$

If the scattering observed is of the form described by eq 6 , then 345 at low $q$, a linear behavior should be obtained in a $\ln \left[q I_{\mathrm{A}}(q)\right] 346$ versus $q^{2}$ representation. This is indeed the case as can be seen 347 from Figure 4D. The cross-section radius of gyration, $R_{\sigma}$, of the 348 

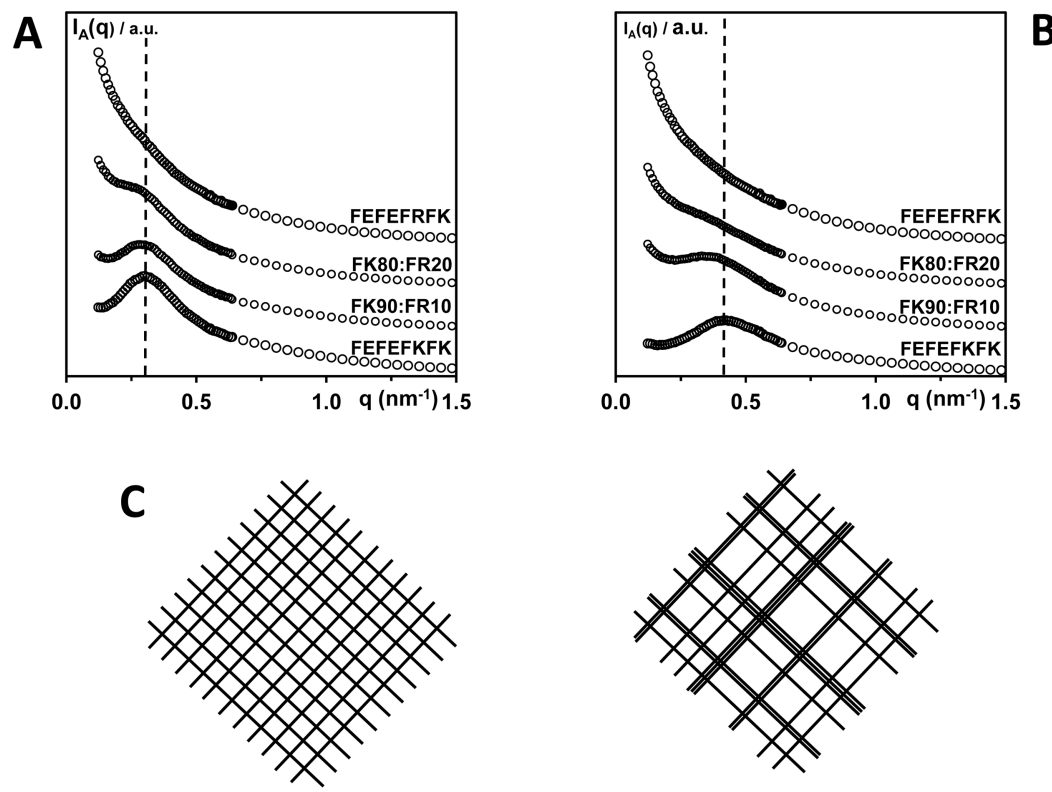

Homogeneous network Uniform fibres

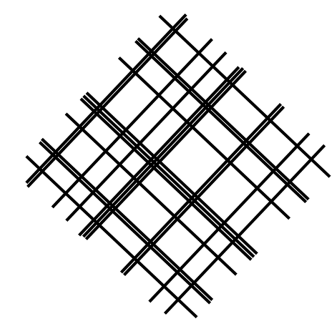

Heterogeneous network

Associated fibres

Figure 5. (A, B) SANS scattering pattern $\left(I_{\mathrm{A}}(q)\right.$ vs $q$ ) obtained for samples prepared at $20 \mathrm{mg} \mathrm{mL}^{-1}(\mathrm{~A})$ and $30 \mathrm{mg} \mathrm{mL}^{-1}$ (B), above the CGC of the samples. (C) $2 \mathrm{D}$ idealized representation of the effect of fiber association/bundling on overall network topology.

349 fibers can be estimated from the slope of the linear section. If 350 we assume that the fibers can be modeled by a plain infinitely 351 long cylinder then $R_{\sigma}$ is related to the diameter of the fiber, $d$, 352 through $R_{\sigma}=\sqrt{d^{2} / 8}$. As can be seen from Figure 4, for 353 FEFEFKFK, a $R_{\sigma}$ of $1.1 \pm 0.2 \mathrm{~nm}$ was obtained corresponding 354 to a fiber diameter of $3.1 \pm 0.6 \mathrm{~nm}$, in good agreement with our 355 previous work and the TEM image in Figure 2. For 356 FEFEFRFK, a slightly higher $R_{\sigma}$ is obtained corresponding to 357 a slightly larger fiber diameter of $4.0 \pm 0.5 \mathrm{~nm}$. For FK90:FR10 358 and FK80:FR20, larger $R_{\sigma}$ are obtained corresponding to fiber 359 diameters of $5.6 \pm 0.6$ and $7.1 \pm 0.8 \mathrm{~nm}$, respectively. From the 360 FTIR and TEM results above, we can assume that the same 361 basic $\beta$-sheet fibers are formed for all the systems, and 362 therefore, the increase in fiber sizes below the CGC suggests 363 the formation of fiber bundles through lateral fiber association. 364 Indeed, it should be kept in mind that the scattering observed is 365 the average scattering of all the rod-like objects, in this case, 366 fibers and fiber bundles, present in the sample. Although the 367 FEFEFRFK sample contains a larger nominal amount of 368 arginine residues (12.5\% of all residues are $\mathrm{R}$ in this sample 369 compared to 5\% for FK80:FR20 and 2.5\% for FK90:FR10), this 370 system shows a "lower" drive to fiber association/bundling. 371 These results suggest that the localization of two $\mathrm{R}$ on the same 372 peptide promotes strong fiber association and bundle 373 formation.

374 In order to investigate the samples morphology, a second 375 series of SANS experiments were carried out above the CGC, at 37620 and $30 \mathrm{mg} \mathrm{mL}^{-1}$. In this regime, the scattering observed is 377 due to the overall sample morphology, that is, fibers and 378 network. The results presented in Figure 5A,B confirm that the 379 introduction of $\mathrm{R}$ affects the morphology of the sample.

380 For FEFEFKFK, a scattering peak is observed at 0.3 and 0.4 $381 \mathrm{~nm}^{-1}$ for the 20 and $30 \mathrm{mg} \mathrm{mL}^{-1}$ samples, respectively. The 382 presence of such a peak suggests that the samples' morphology 383 is highly homogeneous and that a characteristic density 384 fluctuation length is present across the sample. This density fluctuation length is related to the characteristic length scale of 385 the morphology which in turn is related to the characteristic 386 length scales of the two phases forming it: the low density 387 phase (water rich phase, i.e., mesh size), and the high density 388 phase (fiber rich phase: i.e., fiber cross-section). ${ }^{50}$ As a result, if 389 it is assumed that the fiber cross-section is significantly smaller 390 than the network mesh size, the position of the scattering peak 391 can be related through Bragg's law, $(2 \pi) / q$ to the network mesh 392 size $\sim 20$ and $15 \mathrm{~nm}$ for the 20 and $30 \mathrm{mg} \mathrm{mL}^{-1}$ samples, 393 respectively. As expected, the mesh size decreases with 394 increasing peptide concentration as the density of fibers 395 increases. It should be kept in mind that, as discussed above, 396 for FEFEFKFK, no fiber association/aggregation is expected. In 397 addition, at $\mathrm{pH} 3$, this peptide and therefore the fibers carry a 398 positive charge resulting in fiber-fiber repulsion. We 399 hypothesize that as a result there is the formation of highly 400 homogeneous fibers that "avoid" contact due to electrostatic 401 repulsion, a highly homogeneous network and morphology is 402 obtained with branching points and entanglements (no fiber 403 association/bundling).

When FEFEFRFR is introduced (FK90:FR10 and 405 FK80:FR20 samples), the peak scattering intensity decreases, 406 while the scattering intensity at low $q$ increases. These changes 407 in scattering profiles suggest that the sample morphology 408 becomes less homogeneous and that larger scattering entities 409 form, respectively. As mentioned above, the scattering observed 410 in this regime originates from the overall sample morphology, 411 fibers and network; therefore, the increase of the scattering 412 intensity at low $q$ is thought to be related to the formation of 413 fiber bundles as well as to the resulting increase in network 414 mesh size (Figure 5C). It is interesting to note that the changes 415 in scattering patterns are more pronounced at higher 416 concentration. The drive of fiber association is indeed expected 417 to increase with increasing concentration as the fibers are in 418 closer proximity. These results seem to be supported by the 419 TEM images (Figure 4B) showing a more coarse network 420 topology for FK20:FR80 sample, although it should be kept in 421 
422 mind that TEM images can be misleading as the samples are 423 subject to significant conditioning (see Materials and Methods) 424 and the images give a $2 \mathrm{D}$ representation of a $3 \mathrm{D}$ network. 425 For the FEFEFRFK sample, a small shoulder can be 426 observed at $20 \mathrm{mg} \mathrm{mL}^{-1}$, while at $30 \mathrm{mg} \mathrm{mL}^{-1}$, the scattering 427 peak has disappeared altogether. For this sample, too, scattering 428 at low $q$ is observed to increase significantly again pointing 429 toward the formation of larger scattering entities and a decrease 430 in morphology homogeneity. The loss of morphological 431 homogeneity, while fiber bundles form, is not surprising. 432 Indeed, fiber lateral association as occurring here is not a 433 controlled process, and therefore, fiber bundles of different 434 sizes are expected to form. This is expected to lead to a 435 polydispersed mesh size and diffuse scattering (Figure 5). 436 Interestingly, the transparent appearance of the hydrogel 437 formed with FEFEFRFK suggests that any morphological 438 heterogeneity formed is below the half-micron size above where 439 light scattering is observed. On the other hand, FK90:FR10 and 440 FK80:FR20 form slightly hazy hydrogels, suggesting in this case 441 morphological heterogeneities reach the half-micron scale. 442 These observations suggest that the introduction of only one $\mathrm{R}$ 443 on all the peptides leads to more "controlled" fiber association/ 444 aggregation, resulting in fiber bundles of limited size. On the 445 other hand, introduction of FEFEFRFR leads to uncontrolled 446 fiber association/aggregation, resulting in the formation of large 447 bundles and, as discussed above, for pure FEFEFRFR sample to 448 their precipitation.

449 As expected, the change in network topology has a strong 450 effect on the mechanical properties of the hydrogels. In Figure 4516 , the dynamic storage shear moduli obtained for the 3 samples

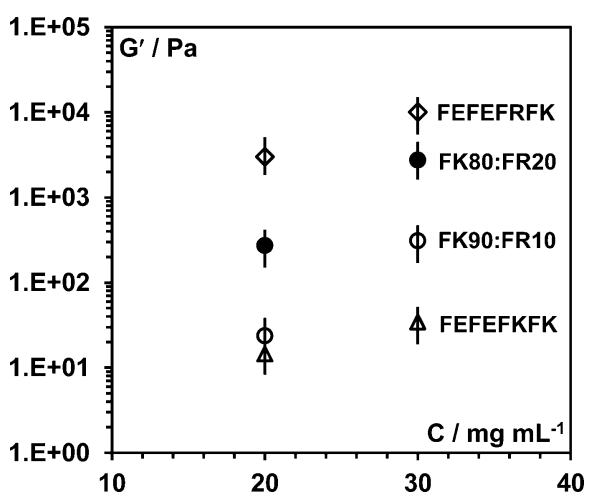

Figure 6. Dynamic shear storage moduli of hydrogels as a function of sample concentration.

452 at 20 and $30 \mathrm{mg} \mathrm{mL}^{-1}$ are presented. The introduction of 453 FEFEFRFR results in a gradual increase in the $G^{\prime}$ of the 454 sample. Surprisingly, FEFEFRFK forms stronger hydrogel at 455 both concentrations, although as discussed above, the fiber 456 association drive seems to be "weaker" for this sample. This 457 clearly points out that in order to control the properties of the 458 hydrogel the fiber association process needs to occur in a 459 controlled fashion. Indeed, the bulk properties of the hydrogels 460 will be a combination between the intrinsic properties of the 461 fibers/fiber bundles and the overall properties of the network, 462 which will depend on the mesh size and therefore on the level 463 of fiber bundling. These results though clearly point out the 464 importance of network topology in controlling the properties of 465 this type of hydrogel.

\section{CONCLUSIONS}

466

We have investigated how network topology affects the 467 mechanical properties of a family of $\beta$-sheet forming peptides 468 whose design is based on the alternation of hydrophobic and 469 hydrophilic residues. Our results clearly show that, in addition 470 to the fiber intrinsic properties, the way these fibers self- 471 assemble themselves to form a $3 \mathrm{D}$ percolated network has a 472 significant impact on the final macroscopic properties of the 473 resulting hydrogels. Taking advantage of the peptide design 474 features used here, we have shown how, by design, in this 475 specific case, introducing a highly interacting hydrophilic amino 476 acid arginine, the fiber-fiber interaction can be manipulated to 477 control the level of fiber lateral association/bundling. This 478 approach allows carefully engineering by design not only the 479 type of fiber formed, in our case, antiparallel $\beta$-sheet, but also 480 the type of network topology formed, branched versus 481 associated. The approach described in this work will be key 482 for the design of hydrogels, exploiting $\beta$-sheet forming peptides 483 with highly controlled mechanical properties, in particular, for 484 the biomedical field.

\section{ASSOCIATED CONTENT}

\section{S Supporting Information}

486

The Supporting Information is available free of charge on the 488 ACS Publications website at DOI: 10.1021/acs.bio- 489 mac.6b01693.

HPLC traces of the four peptides used in this work 491 (PDF).

\section{AUTHOR INFORMATION}

*E-mail: a.saiani@manchester.ac.uk. Phone: +44 (0)161 306495 5981.

ORCID ${ }^{\circ}$

Alberto Saiani: 0000-0002-5504-8306

Present Address

499

${ }^{\S}$ School of Science, Xi'an Jiaotong University, Xi'an, 710049, soo ShaanXi, P.R. China.

Notes

The authors declare no competing financial interest.

\section{ACKNOWLEDGMENTS}

The authors are grateful to the UK Engineering and Physical 505 Sciences Research Council (EPSRC Fellowship Grant No. EP/ 506 K016210/1) and the University of Manchester KTA Pro- 507 gramme for their financial support. J.G. is also grateful to the 508 University Manchester Alumni fund and Peptisyntha (Solvay 509 Group) for funding her scholarship. The authors are also 510 grateful to the Forshungszentrum Jülich (FRJ-2) staff on 511 diffractometer KWS-2 for their support. All research data 512 supporting this publication are directly available within this 513 publication.

(1) Busseron, E.; Ruff, Y.; Moulin, E.; Giuseppone, N. Supra- 516 molecular self-assemblies as functional nanomaterials. Nanoscale 2013, 517 5 (16), 7098-7140.

(2) Stephanopoulos, N.; Ortony, J. H.; Stupp, S. I. Self-assembly for 519 the synthesis of functional biomaterials. Acta Mater. 2013, 61 (3), 520 912-930. 
522 (3) Stupp, S. I.; Palmer, L. C. Supramolecular Chemistry and Self523 Assembly in Organic Materials Design. Chem. Mater. 2014, 26 (1), 524 507-518.

525 (4) Zhang, S. G. Fabrication of novel biomaterials through molecular 526 self-assembly. Nat. Biotechnol. 2003, 21 (10), 1171-1178.

527 (5) De Santis, E.; Ryadnov, M. G. Peptide self-assembly for 528 nanomaterials: the old new kid on the block. Chem. Soc. Rev. 2015, 52944 (22), 8288-8300.

530 (6) Hamley, I. W. Peptide fibrillization. Angew. Chem., Int. Ed. 2007, 53146 (43), 8128-8147.

532 (7) Zhang, S. G. Emerging biological materials through molecular 533 self-assembly. Biotechnol. Adv. 2002, 20 (5-6), 321-339.

534 (8) Pugliese, R.; Gelain, F. Peptidic Biomaterials: From Self535 Assembling to Regenerative Medicine. Trends Biotechnol. 2016, 536 DOI: $10.1016 /$ j.tibtech.2016.09.004.

537 (9) Koutsopoulos, S. Self-assembling peptide nanofiber hydrogels in 538 tissue engineering and regenerative medicine: Progress, design 539 guidelines, and applications. J. Biomed. Mater. Res., Part A 2016, 104 540 (4), 1002-1016.

541 (10) Worthington, P.; Pochan, D. J.; Langhans, S. A. Peptide 542 Hydrogels - Versatile Matrices for 3D Cell Culture in Cancer 543 Medicine. Front. Oncol. 2015, 5 (92), n/a.

544 (11) Castillo Diaz, L. A.; Elsawy, M.; Saiani, A.; Gough, J. E.; Miller, 545 A. F. Osteogenic differentiation of human mesenchymal stem cells 546 promotes mineralization within a biodegradable peptide hydrogel. J. 547 Tissue Eng. 2016, 7, n/a.

548 (12) Diana, M. L.; Eugen, B.; Geoffrey, J. P.; Aikaterini, L. Peptide 549 Self-Assemblies for Drug Delivery. Curr. Top. Med. Chem. 2015, 15 550 (22), 2277-2289.

551 (13) Zhiqiang, Y.; Quan, X.; Chenbo, D.; Su Seong, L.; Liqian, G.; 552 Yiwen, L.; Mathew, D.; Ortenzio; Jun, W. Self-Assembling Peptide 553 Nanofibrous Hydrogel as a Versatile Drug Delivery Platform. Curr. 554 Pharm. Des. 2015, 21 (29), 4342-4354.

555 (14) Zhang, S. G.; Holmes, T.; Lockshin, C.; Rich, A. Spontaneous 556 Assembly of a Self-Complementary Oligopeptide to Form a Stable 557 Macroscopic Membrane. Proc. Natl. Acad. Sci. U. S. A. 1993, 90 (8), 558 3334-3338.

559 (15) Leon, E. J.; Verma, N.; Zhang, S. G.; Lauffenburger, D. A.; $560 \mathrm{Kamm}, \mathrm{R}$. D. Mechanical properties of a self-assembling oligopeptide 561 matrix. J. Biomater. Sci., Polym. Ed. 1998, 9 (3), 297-312.

562 (16) Zhang, S. G.; Altman, M. Peptide self-assembly in functional 563 polymer science and engineering. React. Funct. Polym. 1999, 41 (1-3), $56491-102$.

565 (17) Saiani, A.; Mohammed, A.; Frielinghaus, H.; Collins, R.; 566 Hodson, N.; Kielty, C. M.; Sherratt, M. J.; Miller, A. F. Self-assembly 567 and gelation properties of alpha-helix versus beta-sheet forming 568 peptides. Soft Matter 2009, 5 (1), 193-202.

569 (18) Maslovskis, A.; Guilbaud, J. B.; Grillo, I.; Hodson, N.; Miller, A. 570 F.; Saiani, A. Self-Assembling Peptide/Thermoresponsive Polymer 571 Composite Hydrogels: Effect of Peptideâ€"Polymer Interactions on 572 Hydrogel Properties. Langmuir 2014, 30 (34), 10471-10480.

573 (19) Elsawy, M. A.; Smith, A. M.; Hodson, N.; Squires, A.; Miller, A. 574 F.; Saiani, A. Modification of $\beta$-Sheet Forming Peptide Hydrophobic 575 Face: Effect on Self-Assembly and Gelation. Langmuir 2016, 32 (19), 576 4917-4923.

577 (20) Bowerman, C. J.; Nilsson, B. L. Review self-assembly of 578 amphipathic beta-sheet peptides: Insights and applications. Biopolymers 579 2012, 98 (3), 169-184.

580 (21) Holmes, T. C.; de Lacalle, S.; Su, X.; Liu, G. S.; Rich, A.; Zhang, 581 S. G. Extensive neurite outgrowth and active synapse formation on 582 self-assembling peptide scaffolds. Proc. Natl. Acad. Sci. U. S. A. 2000, 97 583 (12), 6728-6733.

584 (22) Sun, Y.; Li, W.; Wu, X.; Zhang, N.; Zhang, Y.; Ouyang, S.; Song, 585 X.; Fang, X.; Seeram, R.; Xue, W.; He, L.; Wu, W. Functional Self586 Assembling Peptide Nanofiber Hydrogels Designed for Nerve 587 Degeneration. ACS Appl. Mater. Interfaces 2016, 8 (3), 2348-2359.

588 (23) Mujeeb, A.; Miller, A. F.; Saiani, A.; Gough, J. E. Self-assembled 589 octapeptide scaffolds for in vitro chondrocyte culture. Acta Biomater. 590 2013, 9 (1), 4609-4617.
(24) Kisiday, J.; Jin, M.; Kurz, B.; Hung, H.; Semino, C.; Zhang, S.; 591 Grodzinsky, A. J. Self-assembling peptide hydrogel fosters chondrocyte 592 extracellular matrix production and cell division: Implications for 593 cartilage tissue repair. Proc. Natl. Acad. Sci. U. S. A. 2002, 99 (15), 594 9996-10001.

595

(25) Yan, C.; Mackay, M. E.; Czymmek, K.; Nagarkar, R. P.; 596 Schneider, J. P.; Pochan, D. J. Injectable Solid Peptide Hydrogel as a 597 Cell Carrier: Effects of Shear Flow on Hydrogels and Cell Payload. 598 Langmuir 2012, 28 (14), 6076-6087.

599

(26) Castillo Diaz, L. A.; Saiani, A.; Gough, J. E.; Miller, A. F. Human 600 osteoblasts within soft peptide hydrogels promote mineralisation in 601 vitro. J. Tissue Eng. 2014, 5, 2041731414539344.

602

(27) Bradshaw, M.; Ho, D. W.; Fear, M. W.; Gelain, F.; Wood, F. M.; 603 Iyer, S. Designer self-assembling hydrogel scaffolds can impact skin cell 604 proliferation and migration. Sci. Rep. 2014, 4, 6903.

(28) Roberts, D.; Rochas, C.; Saiani, A.; Miller, A. F. Effect of Peptide 606 and Guest Charge on the Structural, Mechanical and Release 607 Properties of beta-Sheet Forming Peptides. Langmuir 2012, 28 (46), 608 16196-16206.

609

(29) Tang, C.; Miller, A. F.; Saiani, A. Peptide hydrogels as 610 mucoadhesives for local drug delivery. Int. J. Pharm. 2014, 465, 427- 611 435.

(30) Branco, M. C.; Pochan, D. J.; Wagner, N. J.; Schneider, J. P. 613 Macromolecular diffusion and release from self-assembled beta-hairpin 614 peptide hydrogels. Biomaterials 2009, 30 (7), 1339-1347. 615

(31) Lindsey, S.; Piatt, J. H.; Worthington, P.; SÃ Inmez, C.; Satheye, 616 S.; Schneider, J. P.; Pochan, D. J.; Langhans, S. A. Beta Hairpin Peptide 617 Hydrogels as an Injectable Solid Vehicle for Neurotrophic Growth 618 Factor Delivery. Biomacromolecules 2015, 16 (9), 2672-2683. 619

(32) Hwang, W. M.; Marini, D. M.; Kamm, R. D.; Zhang, S. Q. 620 Supramolecular structure of helical ribbons self-assembled from a beta- 621 sheet peptide. J. Chem. Phys. 2003, 118 (1), 389-397. 622

(33) Davies, R. P. W.; Aggeli, A.; Beevers, A. J.; Boden, N.; Carrick, 623 L. M.; Fishwick, C. W. G.; McLeish, T. C. B.; Nyrkova, I.; Semenov, A. 624 N. Self-assembling beta-sheet tape forming peptides. Supramol. Chem. 625 2006, 18 (5), 435-443.

626

(34) Lee, N. R.; Bowerman, C. J.; Nilsson, B. L. Sequence length 627 determinants for self-assembly of amphipathic beta-sheet peptides. 628 Biopolymers 2013, 100 (6), 738-50.

629

(35) Lee, N. R.; Bowerman, C. J.; Nilsson, B. L. Effects of Varied 630 Sequence Pattern on the Self-Assembly of Amphipathic Peptides. 631 Biomacromolecules 2013, 14 (9), 3267-3277.

(36) Gallivan, J. P.; Dougherty, D. A. Cation-pi interactions in 633 structural biology. Proc. Natl. Acad. Sci. U. S. A. 1999, 96 (17), 9459- 634 9464.

(37) Schneider, C. P.; Shukla, D.; Trout, B. L. Arginine and the 636 Hofmeister Series: The Role of Ion-Ion Interactions in Protein 637 Aggregation Suppression. J. Phys. Chem. B 2011, 115 (22), 7447- 638 7458.

(38) Vondrek, J. Ã.; Mason, P. E.; Heyda, J.; Collins, K. D.; 640 Jungwirth, P. The Molecular Origin of Like-Charge Arginine-Arginine 641 Pairing in Water. J. Phys. Chem. B 2009, 113 (27), 9041-9045. 642

(39) Higgins, J. S.; Benoit, H. C. Polymer and Neutron Scattering; 643 Clarendon Press: Oxford, 1994.

(40) Roe, R.-J. Methods of X-Ray and Neutron Scattering in Polymer 645 Science; Oxford University Press: New York, 2000.

(41) Guinier, A.; Fournet, G. Small-Angle Scattering of X-rays; John 647 Wiley \& Sons, Inc.: New York, 1955.

(42) Branco, M. C.; Nettesheim, F.; Pochan, D. J.; Schneider, J. P.; 649 Wagner, N. J. Fast Dynamics of Semiflexible Chain Networks of Self- 650 Assembled Peptides. Biomacromolecules 2009, 10 (6), 1374-1380. 651

(43) Caplan, M. R.; Moore, P. N.; Zhang, S. G.; Kamm, R. D.; 652 Lauffenburger, D. A. Self-assembly of a beta-sheet protein governed by 653 relief of electrostatic repulsion relative to van der Waals attraction. 654 Biomacromolecules 2000, 1 (4), 627-631.

655

(44) Boothroyd, S.; Miller, A. F.; Saiani, A. From fibres to networks 656 using self-assembling peptides. Faraday Discuss. 2013, 166, 195-207. 657 
658 (45) Guenet, J. M. Structure versus rheological properties in fibrillar 659 thermoreversible gels from polymers and biopolymers. J. Rheol. 2000, 66044 (4), 947-960.

661 (46) Jones, J. L.; Marques, C. M. Rigid Polymer Network Models. J. 662 Phys. 1990, 51 (11), 1113-1127.

663 (47) Ramzi, M.; Rochas, C.; Guenet, J. M. Structure-properties 664 relation for agarose thermoreversible gels in binary solvents. 665 Macromolecules 1998, 31 (18), 6106-6111.

666 (48) Guilbaud, J. B.; Saiani, A. Using small angle scattering (SAS) to 667 structurally characterise peptide and protein self-assembled materials. 668 Chem. Soc. Rev. 2011, 40 (3), 1200-1210.

669 (49) Guenet, J.-M. Thermoreversible Gelation of Polymers and 670 Biopolymers; Academic Press: London, 1992.

671 (50) Guilbaud, J.-B.; Saiani, A. Using small angle scattering (SAS) to 672 structurally characterise peptide and protein self-assembled materials. 673 Chem. Soc. Rev. 2011, 40 (3), 1200-1210. 


\section{Controlling self-assembling peptide hydrogel properties through network topology}

Jie Gao ${ }^{1,2, \#}$, Claire Tang ${ }^{1,2}$, Andrew M. Smith ${ }^{1,2}$, Aline F. Miller ${ }^{2,3}$ and Alberto Saiani ${ }^{1,2 *}$

${ }^{1}$ School of Materials, The University of Manchester, Oxford road, Manchester, M13 9PL, UK

${ }^{2}$ Manchester Institute of Biotechnology, The University of Manchester, Oxford road, Manchester, M13 9PL, UK.

${ }^{3}$ School of Chemical Engineering and Analytical Sciences, The University of Manchester, Oxford road, Manchester, M13 9PL, UK

\# Current address: School of Science, Xi'an Jiaotong University, Xi'an, 710049, ShaanXi, P.R. China

* Corresponding author. E-mail: a.saiani@manchester.ac.uk; Phone: +44 (0)161 3065981 
Electronic Supplementary Information:
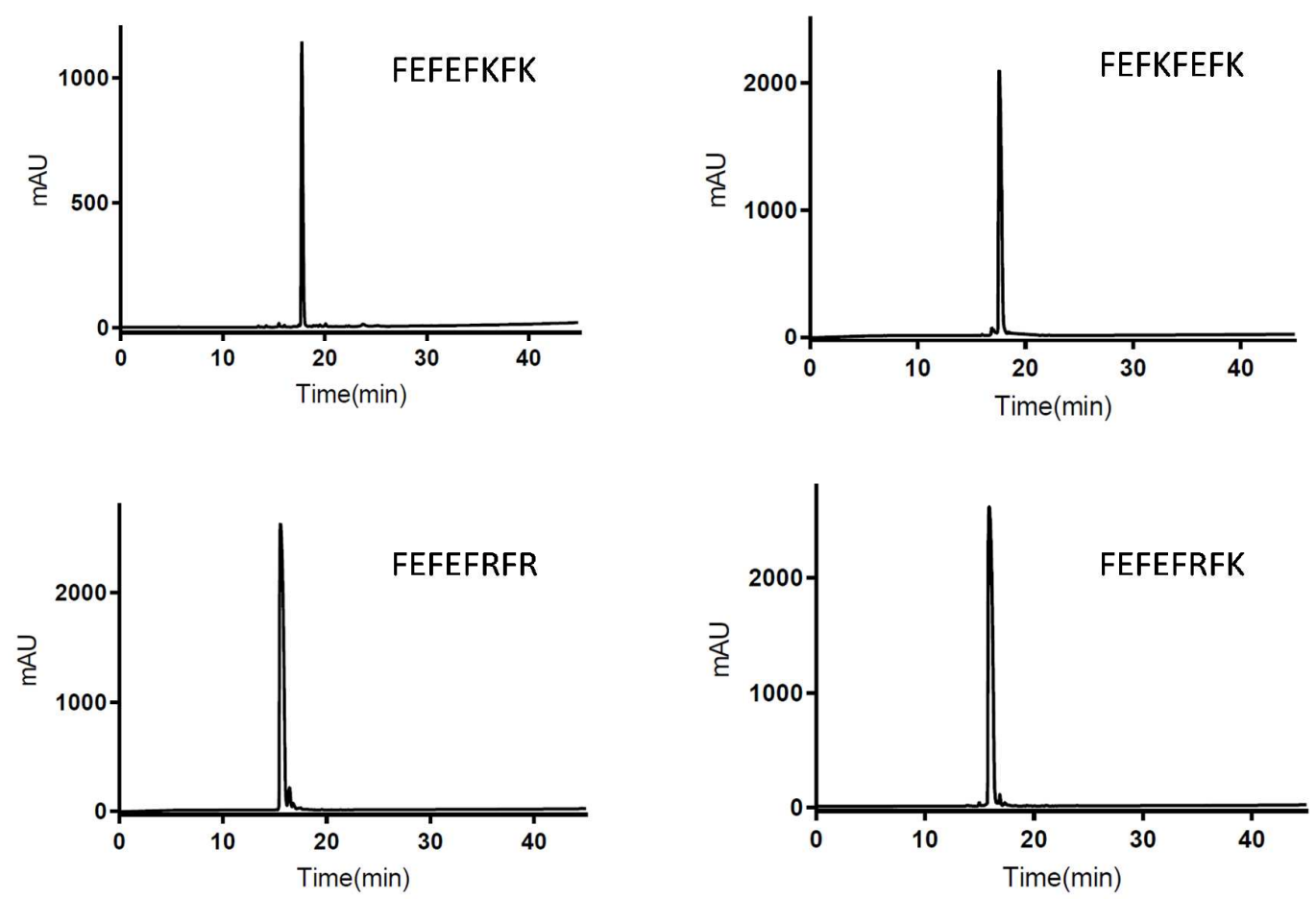

Figure ESI 1: HPLC traces of the four peptides used. (Method: The $1 \mathrm{mg} \mathrm{ml}^{-1}$ peptide solutions in $1 \%$ trifluoroacetic acid (TFA) in water/acetonitrile $(50 / 50 \mathrm{~V} / \mathrm{V})$ were injected on the RP-HPLC column Phenomenex Jupiter $4 \mu$ Proteo column $90 \mathrm{~A}^{\circ}(250 \mathrm{x} 4.66 \mathrm{~mm})$ equipped with UV detector $(\lambda$ $220 \mathrm{~nm}$ ). An elution gradient was used with a flow rate of $1 \mathrm{ml} / \mathrm{min}$ that went from $90 \%$ solvent A $\left(0.05 \%\right.$ TFA in $\left.\mathrm{H}_{2} \mathrm{O}\right) / 10 \%$ solvent $\mathrm{B}\left(0.05 \%\right.$ TFA in $\left.\mathrm{CH}_{3} \mathrm{CN}\right)$ to $30 \%$ solvent $\mathrm{A} / 70 \%$ solvent $\mathrm{B}$ in 45 minutes.) 
Biomacromolecules

Msc: bm6b01693

The following graphic will be used for the TOC:
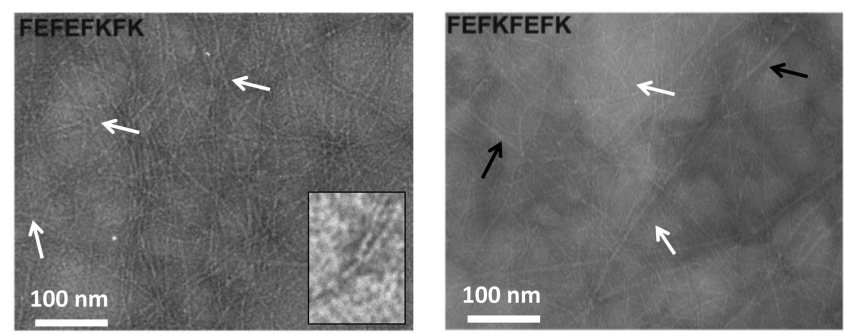